\title{
Leggett-Garg inequality in the context of three flavor neutrino oscillation
}

\author{
Javid Naikoo, ${ }^{*}$ Ashutosh Kumar Alok, ${ }^{\dagger}$ and Subhashish Banerjee \\ Indian Institute of Technology Jodhpur, Jodhpur 342011, India \\ S. Uma Sankar ${ }^{\S}$ \\ Indian Institute of Technology Bombay, Powai 400076, India
}

(Received 14 February 2019; published 3 May 2019)

\begin{abstract}
The present work is devoted to the characterization of the Leggett-Garg inequality (LGI) for three flavored neutrino oscillations in the presence of both matter and charge-conjugation and parity violating effects. This study complements and completes the recent one put forward in arXiv:1710.05562 by relaxing the stationary condition. At variance with the latter case, the LGI contains interference terms which cannot be expressed in terms of experimentally measurable quantities, thus drawing a clear-cut distinction between the two scenarios, as well as highlighting the role of the stationary assumption on such systems. We find that the additional terms are small for a high energy neutrino beam compared to the maximum value attained by the Leggett-Garg parameter.
\end{abstract}

DOI: 10.1103/PhysRevD.99.095001

\section{INTRODUCTION}

One of the most characterizing aspects of quantum mechanics is the principle of superposition, according to which a system exists simultaneously in different states until a measurement is performed on it. A counterintuitive situation occurs when one tries to ascertain the quantum nature of mesoscopic systems, an endeavor that is broadly known as macroscopic quantum coherence (such as, e.g., the famous Schrödinger's cat), which has been subjected to a number of investigations. Bell's inequality [1] represents in this regard a milestone result providing a quantitative criterion to distinguish between classical and quantum correlations between spatially separated systems. In the temporal regime, LeggettGarg inequalities (LGIs) investigate the nature of correlations among measurements performed on the same system but at different instants of time. In [2], the intuition about our view of macroscopic systems was formalized in terms of two principles, namely (i) macroscopic realism and (ii) noninvasive measurability (NIM) [3-5]. The former implies that measurements performed on the system of interest just reveal preexisting values, while the latter (NIM) asserts that such measurements can be performed without changing the state of

\footnotetext{
"naikoo.1@iitj.ac.in

†akalok@iitj.ac.in

subhashish@iitj.ac.in

§uma@phy.iitb.ac.in
}

Published by the American Physical Society under the terms of the Creative Commons Attribution 4.0 International license. Further distribution of this work must maintain attribution to the author(s) and the published article's title, journal citation, and DOI. Funded by SCOAP ${ }^{3}$. the system. Besides providing a test bed for macroscopic coherences, LGI have been employed to investigate the notion of realism, i.e., whether a description of the system under consideration can or cannot be given in terms of a set of hidden parameters. Any violation of LGI would automatically exclude any hidden-variable theory.

Quantum coherences extended over macroscopic distances have been in the spotlight in the context of flavor oscillations in neutrinos and mesons [6-12]. This provides ample reason for promoting such systems as candidates to study LGI. Clearly, in this scenario the postulate of NIM makes contact with experiments somewhat difficult. One then resorts to the additional assumption of stationarity, implying that correlations between different measurements only depend on time differences instead of specific time instants. This leads to a modified version of the LGIs called Leggett-Garg-type inequalities (LGtIs), where all the intermediate nonmeasurable correlations are replaced by measurable ones [13-16]. Such approach was recently used in [17], where the resulting LGtI can be recast in terms of experimentally measurable quantities such as neutrino flavor oscillation probabilities.

In the present work, however, we intend to complement and complete that analysis by considering the full LGI in its original setting and show that it is violated in the context of three flavor neutrino oscillations and matter interactions. It is worth mentioning that various quantum information theoretic quantities were considered in the context of two and three flavor neutrino oscillations also in $[18,19]$. The factorization of the Hilbert space was achieved there by the occupation number of neutrinos introduced in [20]. Here, we study the LGI in the context of three 
flavor neutrino oscillations. A study of the LG-type inequality in the two and three flavor scenario was presented in $[21,22]$ and [23], respectively.

In this contribution we investigate the full three flavor neutrino dynamics, which allows the inclusion of charge parity $(C P)$ violations as well as the disentangling of the mass hierarchy problem. The paper is organized by introducing LG inequalities and the dynamics of neutrinos in vacuum and matter. Then we present the relevant correlators and analyze them generally. We make use of the experimental input parameters like energy and baseline of the two ongoing experiments $\mathrm{NO} \nu \mathrm{A}$ (NuMI Off-axis $\nu_{e}$ Appearance) [24,25] and T2K (Tokai to Kamioka) [26] and the future experiment DUNE (Deep Underground Neutrino Experiment) [27].

\section{LEGGETT-GARG INEQUALITIES IN THREE FLAVOR NEUTRINO OSCILLATIONS}

Here, we give a brief description of LGIs. The time evolution of neutrinos in vacuum and matter is discussed next, followed by a discussion of the LGIs for neutrinos. The next subsection discusses the time evolution of neutrinos in vacuum and matter, followed by the discussion of the Leggett-Garg inequalities for neutrinos with a focus on the two ongoing experiments $\mathrm{NO} \nu \mathrm{A}$ and $\mathrm{T} 2 \mathrm{~K}$ and also the future experiment DUNE.

\section{A. Leggett-Garg inequalities}

Consider a quantum system with an underlying Hilbert space $\mathcal{H}$ and dynamics generated by a Hamiltonian $\mathrm{H}$. Moreover, let $\hat{Q}$ be a generic dichotomic observable (with possible outcomes \pm 1 ) satisfying the properties $\hat{Q}^{\dagger}=\hat{Q}$, $\hat{Q}^{2}=\mathbb{1}$. The two-time correlation function between the measurement of $\hat{Q}$ at times $t_{i}$ and $t_{j}\left(t_{i} \geq t_{j}\right)$ is given by the quantity $C_{i j} \equiv\left\langle\hat{Q}\left(t_{i}\right) \hat{Q}\left(t_{j}\right)\right\rangle$, where $\langle\ldots\rangle$ indicates the average performed over many repetitions. Here $\hat{Q}(t)$ denotes the time evolution of the observable $\hat{Q}$ in the Heisenberg picture, i.e., $\hat{Q}(t) \equiv \hat{U}^{\dagger}(t) \hat{Q} \hat{U}(t)$. For a generic set of $n$ measurements of the dichotomic observable, the LGI provides a clear-cut bound on the parameter $K_{n} \equiv \sum_{i=1}^{n-1} C_{i, i+1}-C_{1, n}$, which allows us to determine the existence of a hidden-variable theory describing the system of interest: For any $n \geq 3$, if realism and NIM are satisfied, then $K_{n} \leq n-2$.

In what follows we will focus on the first of such figures of merit, namely the LG parameter $K_{3}$ and its LG inequality

$$
K_{3}=C_{01}+C_{12}-C_{02} \leq 1
$$

whose violation would provide evidence that a realistic description of the system cannot be given. The quantum mechanical bound for $K_{3}$ is $\frac{3}{2}$ for a two level system [2]. It was shown in [28] that this bound holds for systems with an arbitrary (but finite) number of levels, as long as the measurements are given by just two projectors [29-32]. In the limit $N \rightarrow \infty$, LGI can be violated up to its maximum algebraic sum [33]. The simple form of Eq. (1) is the common feature of Bell-type inequalities which are based on the usual Kolmogorovian rules of the probability. While the two quantities $C_{01}$ and $C_{02}$ can always be easily expressed in terms of measurable quantities, since the first measurement of the observable $\hat{Q}$ occurs at the initial time $t_{0}$, the expression of the intermediate two-time correlation function $C_{12}$ poses in general a real challenge since it depends on the whole history from the initial time $t_{0}$ to the second measurement time $t_{1}$. In order to bypass this difficulty, the NIM postulate is usually replaced by the weaker condition of stationarity. Under the assumption that the time intervals $t_{2}-t_{1}$ and $t_{1}-t_{0}$ are equal to each other, the resulting LGtI then take the much simpler form

$$
\tilde{K}_{3}=2 C_{01}-C_{02},
$$

which again is bounded from above by 1 if realism is valid $[13,14]$. This LGtI was used in the context of neutrino oscillation [17] to address the problem of neutrino mass hierarchy.

In this work we use LGI in its original setting and focus on the ongoing experimental facilities like $\mathrm{NO} \nu \mathrm{A}$ and $\mathrm{T} 2 \mathrm{~K}$ as well as the future DUNE experiment. The matter effect and $C P$ violation are also taken into account in our analysis.

\section{B. Neutrino state evolution in vacuum and in constant matter density}

The nonzero mass squared differences lead to the phenomenon of neutrino oscillation, the existence of a flavor state $\left|\nu_{\alpha}\right\rangle$ into a coherent superposition of mass eigenstates $\left|\nu_{k}\right\rangle$,

$$
\left|\nu_{\alpha}\right\rangle=\sum_{k} U_{\alpha k}^{*}\left|\nu_{k}\right\rangle
$$

where $U_{\alpha k}$ are the elements of a $3 \times 3$ unitary PMNS (Pontecorvo-Maki-Nakagawa-Sakata) mixing matrix $U$ parametrized by three mixing angles $\left(\theta_{12}, \theta_{23}, \theta_{13}\right)$ and a $C P$-violating phase $\delta$. A convenient parametrization for $U\left(\theta_{12}, \theta_{23}, \theta_{32}, \delta\right)$ is given by

$$
U\left(\theta_{12}, \theta_{23}, \theta_{32}, \delta\right)=\left(\begin{array}{ccc}
c_{12} c_{13} & s_{12} c_{13} & s_{23} e^{-i \delta} \\
-s_{12} c_{23}-c_{12} s_{23} s_{13} e^{i \delta} & c_{12} c_{23}-s_{12} s_{23} s_{13} e^{i \delta} & s_{23} c_{13} \\
s_{13} s_{23}-c_{12} c_{23} s_{13} e^{i \delta} & -c_{12} s_{23}-s_{12} c_{23} s_{13} e^{i \delta} & c_{23} c_{13}
\end{array}\right)
$$


where $c_{i j}=\cos \theta_{i j}, s_{i j}=\sin \theta_{i j}, \theta_{i j}$ being the mixing angles and $\delta$ the $C P$-violating phase. The experimental values for the PMNS mixing matrix are taken from the Particle Data Group [34]. Equation (3) represents the state of the neutrino at time $t=0$. At a later time $t$, the flavor state evolves into

$$
\begin{aligned}
\left|\nu_{\alpha}(t)\right\rangle & =\sum_{k} U_{\alpha k}^{*} e^{-i E_{k} t}\left|\nu_{k}\right\rangle, \\
& =\sum_{\beta} \mathcal{A}_{\nu_{\alpha} \rightarrow \nu_{\beta}}(t)\left|\nu_{\beta}\right\rangle,
\end{aligned}
$$

where we have expanded $\left|\nu_{\alpha}\right\rangle$ in terms of the energy (mass) eigenstates $\left|\nu_{k}\right\rangle$, which evolve independently under the Schrödinger equation. This leads to the amplitudes $\mathcal{A}_{\nu_{\alpha} \rightarrow \nu_{\beta}}$ of transition from flavor $\nu_{\alpha}$ to $\nu_{\beta}$ given by

$$
\mathcal{A}_{\nu_{\alpha} \rightarrow \nu_{\beta}}(t)=\sum_{k} U_{\beta k} e^{-i E_{k} t} U_{\alpha k}^{*}
$$

Consequently, the probability of transition at $t \approx L$ is given by

$$
P_{\nu_{\alpha} \rightarrow \nu_{\beta}}(t)=\left|\mathcal{A}_{\nu_{\alpha} \rightarrow \nu_{\beta}}(t)\right|^{2}=\left|\sum_{k} U_{\beta k} e^{-i E_{k} t} U_{\alpha k}^{*}\right|^{2} .
$$

The amplitudes $\mathcal{A}_{\nu_{\alpha} \rightarrow \nu_{\beta}}(t)$ form the elements of the so-called flavor evolution matrix $U_{f}(t)$. In matrix notation the state represented by the vector $\nu_{\alpha}(t) \equiv\left(\nu_{e}(t) \nu_{\mu}(t) \nu_{\tau}(t)\right)$ is connected to the state at $t=0$ by

$$
\boldsymbol{\nu}_{\alpha}(t)=U_{f}(t) \boldsymbol{\nu}_{\alpha}(0)
$$

Neutrinos propagating through a constant matter density (with electron density $N_{e}$ ) interact weakly with electrons. This interaction is characterized by the matter density parameter $A= \pm \sqrt{2} G_{F} N_{e}$. As a result of this interaction, the Hamiltonian $H_{m}=\operatorname{diag}\left[E_{1}, E_{2}, E_{3}\right]$ (in mass basis) picks up an interaction term $V_{f}=\operatorname{diag}[A, 0,0]$ (in flavor basis). This leads to the following form of the flavor evolution matrix [35]

$$
U_{f}(L)=\phi \sum_{n=1}^{3} \frac{e^{-i \lambda_{n} L}}{3 \lambda_{n}^{2}+c_{1}}\left[\left(\lambda_{n}^{2}+c_{1}\right) \mathbf{I}+\lambda_{n} \tilde{T}+\tilde{T}^{2}\right] .
$$

The phase $\phi=e^{-i \frac{T \cdot \mathcal{H}_{m}}{3} L}, \quad c_{1}=\operatorname{det}(T) \operatorname{tr}\left(T^{-1}\right)$ and the Hamiltonian in mass basis is $\mathcal{H}_{m}=H_{m}+U^{-1} V_{f} U$. The $\lambda_{n}$ are the eigenvalues of $T$ and the matrix $T$ and $\tilde{T}$ are given in [35]. The flavor evolution operator defined in Eq. (9) can be used to deal with the situation when neutrinos pass through a series of matter densities with the matter density parameters $A_{1}, A_{2}, \ldots, A_{n}$. In this case, the total evolution operator becomes

$$
U_{f}^{\mathrm{tot}}(L)=\prod_{i=1}^{n} U_{f}\left(L_{i}\right)
$$

Here, $L=\sum_{i=1}^{n} L_{i}$ and $U_{f}\left(L_{i}\right)$ is evaluated for the density parameter $A_{i}$. A useful application of Eq. (10) has been suggested for the mantle-core-mantle step function model simulating Earth's matter density profile [36].

\section{Leggett-Garg inequality for neutrinos}

In this subsection we characterize the LG parameter $K_{3}$ in the case of three flavor neutrinos and study the validity of LGI using input parameters from two of the current major experimental platforms, namely $\mathrm{NO} \nu \mathrm{A}$ and $\mathrm{T} 2 \mathrm{~K}$ and the future experiment DUNE. Therefore, we focus on having a specific initial flavor eigenstate, i.e., $\nu_{\mu}$, and choose equal time intervals $\left(t_{0}=0, t_{1}=t, t_{2}=2 t\right)$. Herewith, our LG parameter $K_{3}$ becomes the sum of the following correlation functions

$$
K_{3}=C(0, t)+C(t, 2 t)-C(0,2 t) \leq 1 .
$$

To compute the two-time correlation functions $C$ we need to employ the dichotomic observable $\hat{Q}=2\left|\nu_{\alpha}\right\rangle\left\langle\nu_{\alpha}\right|-\mathbb{1}$, which physically corresponds to asking whether the neutrino is still in the state $\left|\nu_{\mu}\right\rangle$ (associated outcome 1) or has undergone a transition to another flavor state $\left|\nu_{\alpha}\right\rangle$ with $\alpha \neq \mu$ (associated outcome -1 ). Straightforwardly one finds for

$$
\begin{aligned}
C(0, t)= & 4 \delta_{\alpha \mu}\left\langle\nu_{\mu}(t) \mid \nu_{\alpha}\right\rangle\left\langle\nu_{\alpha} \mid \nu_{\mu}(t)\right\rangle \\
& -2\left\langle\nu_{\mu}(t) \mid \nu_{\alpha}\right\rangle\left\langle\nu_{\alpha} \mid \nu_{\mu}(t)\right\rangle-2 \delta_{\alpha \mu}+1 \\
= & \begin{cases}2 \mathcal{P}_{\mu \rightarrow \mu}(t)-1 & \text { for } \alpha=\mu \\
1-2 \mathcal{P}_{\mu \rightarrow \alpha}(t) & \text { for } \alpha \neq \mu\end{cases}
\end{aligned}
$$

where $\mathcal{P}_{\mu \rightarrow \mu}(t)$ is the surviving probability and $\mathcal{P}_{\mu \rightarrow \alpha}(t)$ is the transition probability. Use has been made of the fact that the completeness condition in three flavor neutrino oscillation is $\sum_{\alpha=e, \mu, \tau}\left|\nu_{\alpha}\right\rangle\left\langle\nu_{\alpha}\right|=\mathbb{1}$, leading to $P_{\mu e}(t)+$ $P_{\mu \mu}(t)+P_{\mu \tau}(t)=1$ in Eq. (12).

The probabilities with matter effect depend on the neutrino energy $\mathrm{E}$, the mass square differences $\Delta_{i j}=m_{j}^{2}-m_{i}^{2}$, the matter density parameter $A$, the mixing angles $\theta_{i j}$ and the $C P$-violating phase $\delta$, i.e., $\mathcal{P}_{\mu \rightarrow \alpha}=\mathcal{P}_{\mu \rightarrow \alpha}\left(E, t, A, \Delta_{12}, \Delta_{31}, \theta_{12}, \theta_{23}, \theta_{13}, \delta\right)$. For brevity in nomenclature, we suppress all the other dependences except the time dependence. The ongoing neutrino experiments $\mathrm{NO} \nu \mathrm{A}$ and $\mathrm{T} 2 \mathrm{~K}$ are studying the transition probabilities, $P_{\mu \rightarrow e}(t)$. Thus we focus on the choice $\alpha=e$ in the following.

The tricky part is the correlation $C(t, 2 t)$ since it cannot be rewritten into surviving or/and transition probabilities if one does not invoke the stationary condition, i.e., 

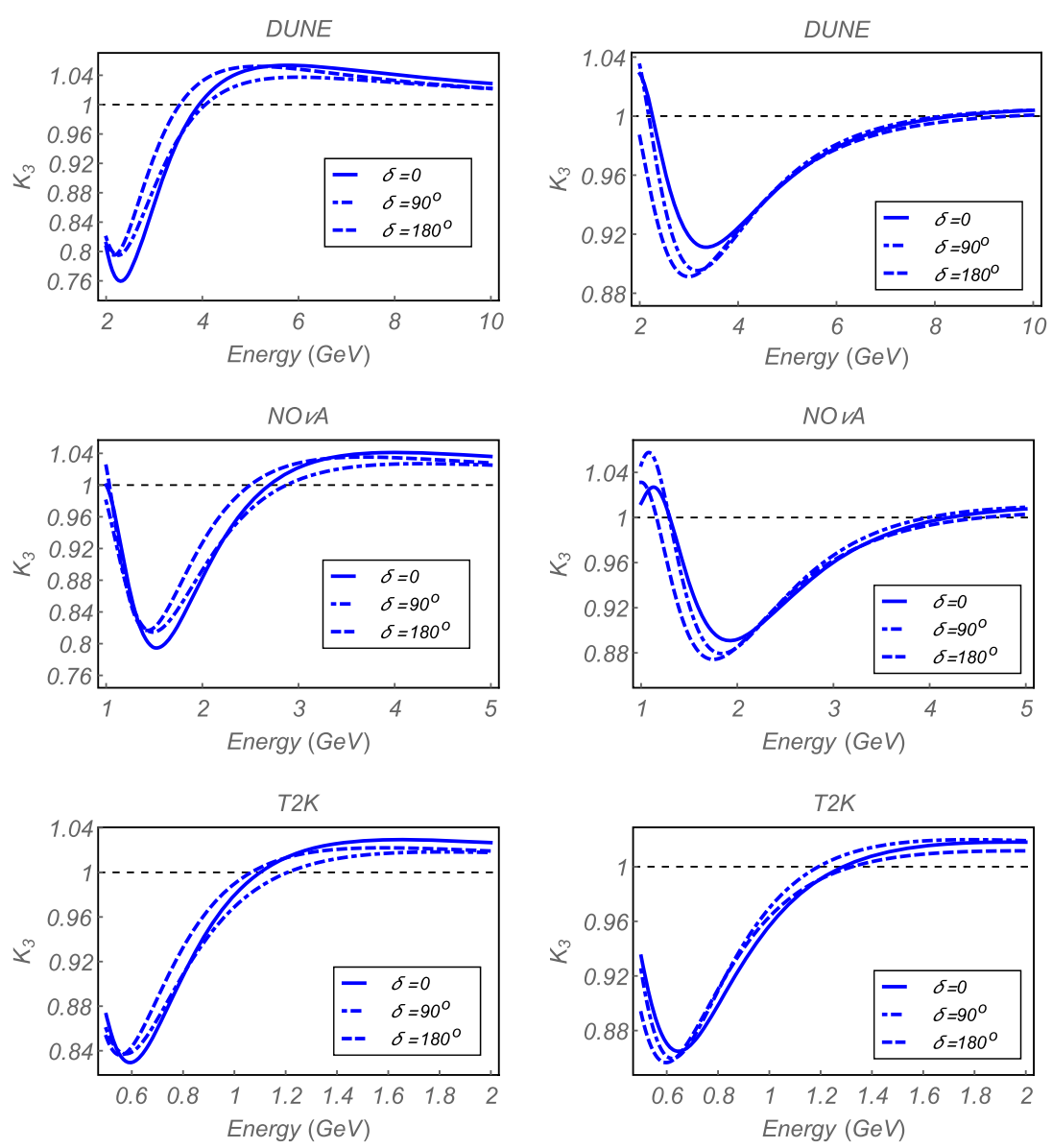

FIG. 1. Leggett-Garg function $K_{3}$ plotted against energy for DUNE (top), NO $\nu$ A (middle) and T2K (bottom) experimental setups for different values of the $C P$-violating phase $\delta$. The left and right panels correspond to the initial neutrino and antineutrino state, respectively. The sign of $\Delta_{31}$ is taken to be positive. The time can be identified with the length (baseline) which is 1300,810 and $295 \mathrm{~km}$ for DUNE, $\mathrm{NO} \nu \mathrm{A}$ and $\mathrm{T} 2 \mathrm{~K}$, respectively.

considering Leggett-Garg-type inequalities which has been done in detail in [17]. The correlation function computes to

$$
\begin{aligned}
C(t, 2 t)= & 1-2 P_{\mu \rightarrow e}(t)-2 P_{\mu \rightarrow e}(2 t) \\
& +4 \alpha(t) P_{\mu \rightarrow e}(2 t)+4 \beta(t) .
\end{aligned}
$$

Finally, our LG function is given by

$$
K_{3}=1-4 P_{\mu \rightarrow e}(t)+4 \alpha(t) P_{\mu \rightarrow e}(2 t)+4 \beta(t),
$$

with

$$
\begin{gathered}
\alpha(t)=\left|U_{f}^{11}(t)\right|^{2}, \\
\beta(t)=\operatorname{Re}\left[U_{f}^{11}(t) \bar{U}_{f}^{21}(t) U_{f}^{22}(2 t) \bar{U}_{f}^{12}(2 t)\right. \\
\left.+U_{f}^{11}(t) \bar{U}_{f}^{31}(t) U_{f}^{32}(2 t) \bar{U}_{f}^{12}(2 t)\right] .
\end{gathered}
$$

Here $\bar{U}_{f}^{i j}=U_{f}^{i j *}$ represents the complex conjugate of $U_{f}^{i j}$, the $i j$ th element of the flavor evolution matrix $U_{f}$ defined in Eq. (8). The parameters $\alpha$ and $\beta$, apart from time $t$, also depend on the energy of neutrino and the mixing angles and mass squared differences. Also $\alpha$, unlike $\beta$, is independent of the $C P$-violating phase $\delta$. It should be noticed that for $\alpha=0.5$ and $\beta=0$, we recover the stationarity limit of LGI given by Eq. (2). An important observation is that for higher energies, the interference term $\beta$ converges to zero. Also, the term $\alpha$, which varies between zero and 1 , averages to $\frac{1}{2}$, thereby taking LGI to LGtI. Therefore, LGtI can be thought of as a kind of LGI for higher neutrino energies.

\section{Leggett-Garg inequality in neutrino experiments}

Neutrino oscillation experiments are typically in the ultrarelativistic limit, and hence $t$ can be approximated by $L$ [37]. The distance traveled by the neutrinos in a given experiment is an important parameter and is called the baseline of the experiment. In this work, we have studied the LGI for two ongoing experiments $\mathrm{NO} \nu \mathrm{A}[38,39]$ and T2K [40,41] and the future experiment DUNE [27]. The baseline for these three experiments is $1300 \mathrm{~km}$ for DUNE, $810 \mathrm{~km}$ for $\mathrm{NO} \nu \mathrm{A}$ and $295 \mathrm{~km}$ for T2K. Both DUNE and 

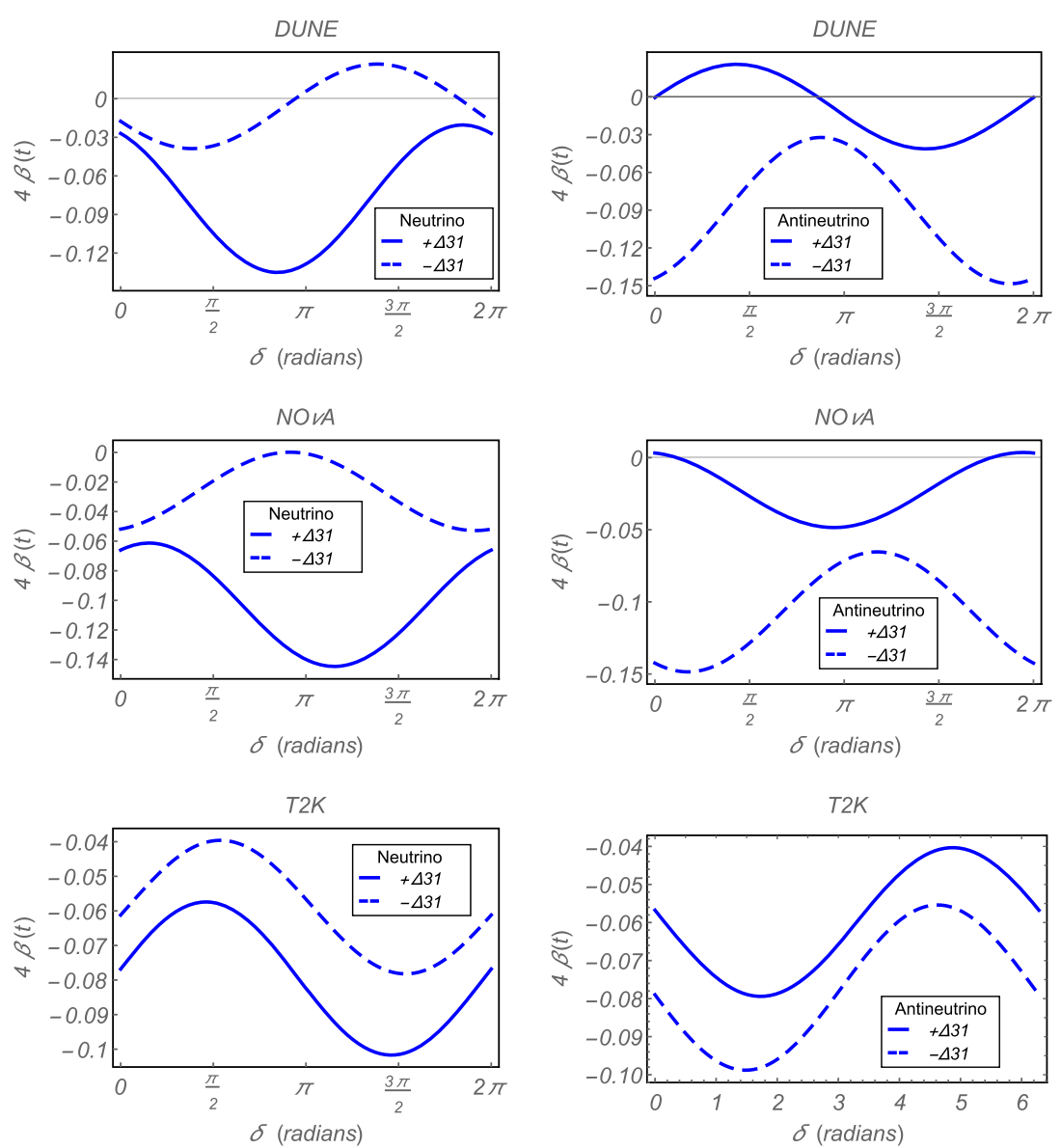

FIG. 2. Plots of the term $4 \beta(t)$, Eq. (14), with respect to $C P$-violating phase $\delta$ for DUNE (left), NO $\nu \mathrm{A}$ (middle) and T2K (right). Solid and dashed curves correspond to the positive and negative signs of $\Delta_{31}$, respectively. The energies used are $3.5 \mathrm{GeV}$ (DUNE), $2.5 \mathrm{GeV}$ $(\mathrm{NO} \nu \mathrm{A})$ and $1.4 \mathrm{GeV}(\mathrm{T} 2 \mathrm{~K})$ and pertain to the maximum neutrino flux in the respective experimental setups. The left and right panels correspond to the case of the neutrino and antineutrino, respectively.

$\mathrm{NO} \nu \mathrm{A}$ use the neutrinos from Fermilab with the energy between 2 and $10 \mathrm{GeV}$ for the former and 1 and $5 \mathrm{GeV}$ for the latter. T2K uses the neutrino source from JPARC (Japan Proton Accelerator Research Complex) in Tokai with the approximate energy $0.5-1 \mathrm{GeV}$. All these experiments use the $\nu_{\mu} / \bar{\nu}_{\mu}$ source and the neutrinos travel the matter density of approximately $2.8 \mathrm{gm} / \mathrm{cm}^{3}$ which corresponds to the density parameter $A \approx 1.01 \times 10^{-13} \mathrm{eV}$.
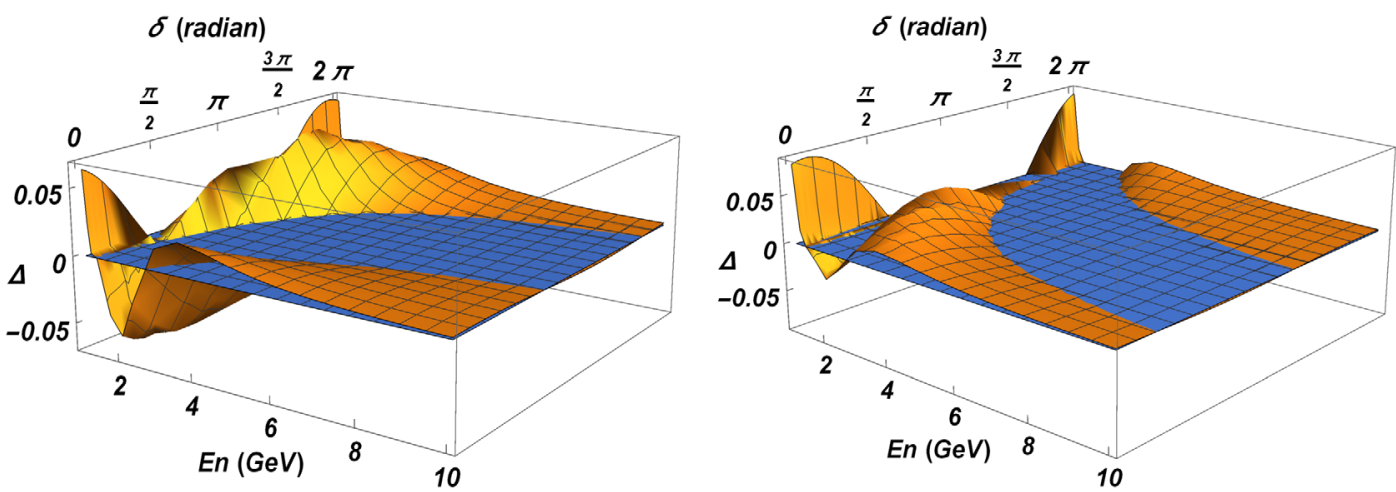

FIG. 3. Showing the difference $\Delta=K_{3}-\tilde{K}_{3}$ as a function of energy $E_{n}$ and $C P$-violating phase $\delta$. The left and right panels correspond to the case of the neutrino and antineutrino, respectively. The function $\tilde{K}_{3}$ and $K_{3}$ are given by Eqs. (2) and (14), respectively. The difference converges to zero for higher energies for all values of $\delta$, implying that one can safely approximate LGI by LGtI for high energy scenarios. This is because the nonmeasurable terms $\alpha$ and $\beta$ in LGI can be approximated by $\frac{1}{2}$ (average value) and 0 (high energy limit), respectively, leading to LGtI. 
In Fig. 1 the LG function $K_{3}$ is depicted with respect to the energy corresponding to these three experimental setups for different $\delta$ values. The behavior of $K_{3}$ is depicted both for the neutrino and antineutrino initial state. The violation is more prominent in the former case, suggesting that the neutrino is a better option for testing LGI if $\Delta_{31}$ is positive. For negative values of $\Delta_{31}$ (not shown here) the violation is more for the antineutrinos [17]. It can be observed from Eq. (14) that due to the presence of the $\alpha(t)$ and $\beta(t)$ terms, $K_{3}$ cannot be expressed only in terms of the neutrino survival and transition probabilities. This term $\beta(t)$ is plotted in Fig. 2 with DUNE, NO $\nu$ A and T2K parameters as a function of the $C P$-violating parameter $\delta$. Let us note that the $\beta(t)$ term is negative for the experimental parameters thus reducing the $K_{3}$ value consequently a possible violation of the bound one. In general, it can be positive as in the case of the DUNE experiment. It turns out that at higher energies, the difference between the LGtI and LGI converges to zero as depicted in Fig. 3. Therefore at higher energies, one can safely approximate LGI by LGtI. One can attribute this to the fact that at higher energies the $\beta$ term goes to zero. Since $0 \leq \alpha \leq 1$, which averages to $\frac{1}{2}$, under these approximations, i.e., $\alpha \approx \frac{1}{2}$ and $\beta \approx 0$, LGI reduces to LGtI.

\section{NIM CONDITION IN NEUTRINO EXPERIMENTS}

The NIM condition renders LGI difficult from the experimental point of view. This feature is captured in Eq. (14) in the form of nonmeasurable terms $\alpha$ and $\beta$ defined in Eqs. (15) and (16), respectively. The NIM condition is intertwined with the LGI as a memory, or to be more precise, the lack of it, that the system has been measured. The concept of stationarity introduced in $[13,14]$ provides a way to bypass the problem. The twotime correlations are functions of the time difference $t_{j}-t_{i}$ and one can replace the term $C(t, 2 t)$, the source of $\alpha$ and $\beta$, by $C(0, t)$ leading to the LG-type inequality Eq. (2) which is completely expressed in terms of the measurable neutrino oscillation probabilities. In our earlier work [17], we used this formalism to address the mass hierarchy problem in neutrino physics. In the present case, one can recover the stationarity limit of LGI under the conditions that $\alpha=0.5$ and $\beta=0$. Since, $0 \leq \alpha \leq 1$, we can think of $\alpha=0.5$ as the average value. Also, the term $\beta \rightarrow 0$ for higher energies. Therefore, LGtI comes out as an average of LGI in high energy regime.

\section{CONCLUSION}

In this work we develop LG inequalities in the context of three flavor neutrino oscillations including matter as well as $C P$-violating effects. It turns out that the $\mathrm{LG}$ function contains nonmeasurable terms, $\alpha$ and $\beta$, apart from the experimentally measurable probabilities. Under the approximations $\alpha \approx 0.5$ and $\beta \approx 0$, one recovers the stationarity limit of the LGI. These approximations hold well in high energy experiments, since the interference term $\beta \rightarrow 0$ at higher energies, and $\alpha$ which varies between 0 and 1 , can be approximated by its average value $\frac{1}{2}$. Therefore, the LGtI comes out as a high energy limit of LGI in the three flavor scenario of neutrino oscillation. Energy and baseline are seen to be the most important factors contributing to the violation.

\section{ACKNOWLEDGMENTS}

We acknowledge useful discussions with B. C. Hiesmayr and G. Guarnieri. This work is partially supported by DST INT/AUSTRIA/BMWF/P-07/2017 Austria Project Based Personnel Exchange Programme. S. B. acknowledges partial financial support from Project No. 03 (1369)/16/EMRII funded by the Council of Scientific \& Industrial Research, New Delhi.
[1] J. S. Bell, Physics (Long Island City, N.Y.) 1, 195 (1964). [2] A. J. Leggett and A. Garg, Phys. Rev. Lett. 54, 857 (1985).

[3] C. Emary, N. Lambert, and F. Nori, Rep. Prog. Phys. 77, 016001 (2014).

[4] J. Kofler and C. Brukner, Phys. Rev. Lett. 101, 090403 (2008).

[5] T. Fritz, New J. Phys. 12, 083055 (2010).

[6] K. Dixit, J. Naikoo, S. Banerjee, and A. K. Alok, Eur. Phys. J. C 78, 914 (2018).

[7] K. Dixit, A. K. Alok, and S. Banerjee, Eur. Phys. J. C 78, 914 (2018).

[8] J. Naikoo, A. K. Alok, and S. Banerjee, Phys. Rev. D 97, 053008 (2018).
[9] J. Naikoo and S. Banerjee, Eur. Phys. J. C 78, 602 (2018).

[10] K. Dixit, J. Naikoo, S. Banerjee, and A. K. Alok, Eur. Phys. J. C 79, 96 (2019).

[11] A. K. Alok, S. Banerjee, and S. Uma Sankar, Phys. Lett. 94B, 749 (2015).

[12] S. Banerjee, A. K. Alok, and R. MacKenzie, Eur. Phys. J. Plus 131, 129 (2016).

[13] S. F. Huelga, T. W. Marshall, and E. Santos, Phys. Rev. A 52, R2497 (1995).

[14] S. F. Huelga, T. W. Marshall, and E. Santos, Phys. Rev. A 54, 1798 (1996).

[15] S. Huelga, T. Marshall, and E. Santos, Europhys. Lett. 38, 249 (1997). 
[16] G. Waldherr, P. Neumann, S. Huelga, F. Jelezko, and J. Wrachtrup, Phys. Rev. Lett. 107, 090401 (2011).

[17] J. Naikoo, A. K. Alok, S. Banerjee, S. U. Sankar, G. Guarnieri, and B. C. Hiesmayr, arXiv:1710.05562.

[18] A. K. Alok, S. Banerjee, and S. U. Sankar, Nucl. Phys. B909, 65 (2016).

[19] S. Banerjee, A. K. Alok, R. Srikanth, and B. C. Hiesmayr, Eur. Phys. J. C 75, 487 (2015).

[20] M. Blasone, F. DellAnno, S. De Siena, and F. Illuminati, Eur. Phys. Lett. 85, 50002 (2009).

[21] J. A. Formaggio, D. I. Kaiser, M. M. Murskyj, and T. E. Weiss, Phys. Rev. Lett. 117, 050402 (2016).

[22] Q. Fu and X. Chen, Eur. Phys. J. C 77, 775 (2017).

[23] D. Gangopadhyay and A. S. Roy, Eur. Phys. J. C 77, 260 (2017).

[24] R. B. Patterson (NOvA Collaboration), Nucl. Phys. B, Proc. Suppl. 235-236, 151 (2013).

[25] P. Adamson et al. (NOvA Collaboration), Phys. Rev. Lett. 116, 151806 (2016).

[26] K. Abe et al. (T2K Collaboration), Nucl. Instrum. Methods Phys. Res., Sect. A 659, 106 (2011).

[27] R. Acciarri et al. (DUNE Collaboration), arXiv:1512 .06148 .

[28] C. Budroni, T. Moroder, M. Kleinmann, and O. Guhne, Phys. Rev. Lett. 111, 020403 (2013).

[29] N. Lambert, R. Johansson, and F. Nori, Phys. Rev. B 84, 245421 (2011).
[30] R. E. George, L. M. Robledo, O. J. Maroney, M. S. Blok, H. Bernien, M. L. Markham, D. J. Twitchen, J. J. Morton, G. A. D. Briggs, and R. Hanson, Proc. Natl. Acad. Sci. U.S.A. 110, 3777 (2013).

[31] J. Kofler and C. Brukner, Phys. Rev. Lett. 99, 180403 (2007).

[32] M. M. Wilde, J. M. McCracken, and A. Mizel, Proc. R. Soc. A 466, 1347 (2010).

[33] C. Budroni and C. Emary, Phys. Rev. Lett. 113, 050401 (2014).

[34] C. Patrignani et al. (Particle Data Group), Chin. Phys. C 40, 100001 (2016).

[35] T. Ohlsson and H. Snellman, J. Math. Phys. (N.Y.) 41, 2768 (2000).

[36] T. Ohlsson and H. Snellman, Phys. Lett. B 474, 153 (2000).

[37] C. Giunti and C. W. Kim, Fundamentals of Neutrino Physics and Astrophysics, (Oxford University Press, Oxford, 2007).

[38] R. B. Patterson (NOfvA Collaboration), Nucl. Phys. B, Proc. Suppl. 235-236, 151 (2013).

[39] P. Adamson et al. (NOvA Collaboration), Phys. Rev. Lett. 116, 151806 (2016).

[40] K. Abe et al. (T2K Collaboration), Phys. Rev. Lett. 112, 061802 (2014).

[41] K. Abe et al. (T2K Collaboration), Phys. Rev. Lett. 111, 211803 (2013). 